\title{
Modeling Antenna Array Elements and Bandwidth Enhanced by Genetic Algorithm
}

\author{
Yu-Hao Liu*, An-Shyi Liu, Yi-Hsin Pang, and Ruey-Beei Wu \\ Department of Electrical Engineering and \\ Graduate Institute of Communication Engineering, \\ National Taiwan University, \\ Taipei, Taiwan, 10617, R. O. C.
}

Email: r90942047@ms90.ntu.edu.tw, rbwu@ew.ee.ntu.edu.tw

\begin{abstract}
A systematic approach for optimal design of broadband antenna arrays is proposed. The method is based on circuit modeling of the antenna array and optimization with Genetic Algorithm. An optimal design of a printed dipole antenna array will be demonstrated.
\end{abstract}

\section{Introduction}

Antenna array usually has the advantages of high gain and high directivity. However, such an antenna usually shows an insufficient impedance bandwidth for satisfying the required operating band. To overcome this problem, a systematical way of searching optimum solution based on Genetic Algorithm[1] for broadband antenna array is proposed in this article.

\section{Antenna Configuration}

Fig. 1 is the schematic drawing of the antenna array comprised of two printed circuits on opposite sides of a thin dielectric substrate of which thickness is $0.5 \mathrm{~mm}$ and relative permittivity $\varepsilon_{r}=3.38$. The antenna array consists of six dipole-like antenna elements, and is used for wireless local area network (WLAN) application at $2.4 \mathrm{GHz}$. For a good impedance matching consideration, an additional tuning microstrip line is placed on the front end. The overall size of antenna is about $6.5 \lambda \times 0.2 \lambda$, where $\lambda$ is the guided wavelength. To accurately predict the performance of this structure by using commercial EM software, such as IE3D, the considerable simulation time raises difficulties in finding optimum solution for impedance bandwidth. An equivalent circuit model must be used for further optimum process.

\section{Equivalent Circuit Model}

As shown in Fig. 2, the antenna array can be considered as two major parts:

This work was supported by the National Science Council, Republic of China, under Grant NSC 91-2622-E-002-050-CC3. 
transmission lines and array elements. The transmission line is regarded as an ideal microstrip line in this work and therefore the modeling of array element is the primary issue. In other words, whether the equivalent circuit performs well or not depends heavily on the model of array element. An intuitive model that ignores the mutual coupling between transmission lines and array elements, and the mutual coupling of array elements is first examined, as shown in Fig. 3. The input impedance of a single element is calculated by the full-wave solver, IE3D, and then is put into the circuit model. The return loss of the circuit model can then be quickly obtained by a circuit simulator, and is plotted in Fig. 4. The return loss of the whole antenna structure analyzed by IE3D is also plotted for comparison. However, these two simulation results are quite different.

The element model is then improved to take the effect of mutual coupling between array elements and transmission lines into consideration. As shown in Fig. 5, the antenna element with two sections of transmission line as a two-port circuit is first anaiyzed, and the input impedance of the antenna element is then extracted and is put into the circuit model. The return loss of this equivalent circuit is depicted in Fig. 6 as compared with the simulation result by IE3D.

The modeling of the antenna element is further improved by taking into account the mutual coupling between array elements. As an illustration, an antenna array consists of three elements is considered, as shown in Fig. 7. The voltage on each node $\mathrm{j}$ and the current flowing into the node are denoted as $\mathrm{V}_{\mathrm{j}}$ and $\mathrm{I}_{\mathrm{j}}$ respectively, where $\mathrm{j}=1,2, \ldots, 5$. The relation of voltages and currents can be written in a matrix form,

$$
[\mathrm{V}]=[\mathrm{Z}] \cdot[\mathrm{I}] .
$$

The self impedances, diagonal terms of the $Z$ matrix, are already obtained in the previous improved model. The mutual impedances are obtained by analyzing the corresponding two elements and an open circuit between them must be assured. In addition, the voltages and currents are coupled by transmission line equation, except for the input port,

$$
\begin{aligned}
& {\left[\begin{array}{c}
\mathrm{V}_{2} \\
\mathrm{~V}_{3}
\end{array}\right]=\left[\begin{array}{ll}
\mathrm{jZ} \mathrm{Z}_{01} \cot \left(\beta l_{1}\right) & \mathrm{j} \mathrm{Z}_{01} \csc \left(\beta l_{1}\right) \\
\mathrm{jZ} \mathrm{Z}_{01} \csc \left(\beta l_{1}\right) & \mathrm{j} \mathrm{Z}_{01} \cot \left(\beta l_{1}\right)
\end{array}\right] \cdot\left[\begin{array}{l}
\mathrm{I}_{2} \\
\mathrm{I}_{3}
\end{array}\right],} \\
& {\left[\begin{array}{c}
\mathrm{V}_{4} \\
\mathrm{~V}_{5}
\end{array}\right]=\left[\begin{array}{ll}
\mathrm{j} \mathrm{Z}_{02} \cot \left(\beta l_{2}\right) & \mathrm{j} \mathrm{Z}_{02} \csc \left(\beta l_{2}\right) \\
\mathrm{jZ} \mathrm{Z}_{02} \csc \left(\beta l_{2}\right) & \mathrm{j} \mathrm{Z}_{02} \cot \left(\beta l_{2}\right)
\end{array}\right] \cdot\left[\begin{array}{l}
\mathrm{I}_{4} \\
\mathrm{I}_{5}
\end{array}\right],}
\end{aligned}
$$

In the above equations, $\beta$ is the propagation constant, $Z_{0 \mathrm{j}}$ and $l_{\mathrm{j}}$ are the characteristic impedance and length of the $\mathrm{j}$-th transmission line respectively, where $j=1,2$. Substitute equations (2a) and (2b) into (1), the input impedance $V_{1} / I_{1}$ can then be obtained and shows good consistency with the one obtained by Ensemble in the IEEE 802.11 b frequency band $(2.4-2.4835 \mathrm{GHz})$ as shown 
in Fig. 8. However, the CPU time for circuit simulation is negligible as compare to the full-wave simulation.

\section{Optimum Process}

A simple program to calculate the antenna input impedance in the final equivalent circuit model is developed. Applying Genetic algorithm, an optimal design is proposed for maximum bandwidth. The characteristic impedances of the transmission lines, and the width and length of the tuning microstrip line, are set to be parameters in GA optimization. Fig. 9 is the return loss of the optimal design and shows that the bandwidth is about three times wider than that of the original antenna array design.

\section{Conclusion}

We have proposed a systematical way of searching optimum solution based on Genetic Algorithm for broadband antenna array.

\section{Acknowledgement}

The author would like to thank EVERFOCUS for fabricating the antennas.

\section{Reference}

[1] Haupt, R.L., "An introduction to genetic algorithms for electromagnetics," IEEE Antennas and Propagation Magazine, Vol. 37, No. 2, Apr. 1995, pp. $7-15$.

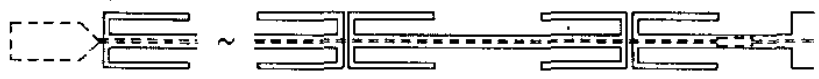

Fig. 1

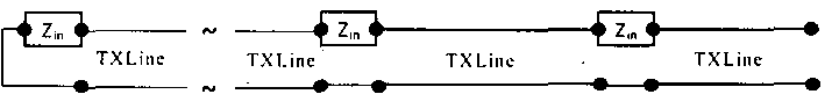

Fig. 2 


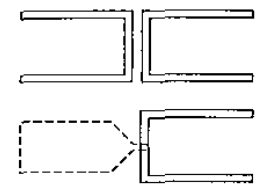

Fig. 3

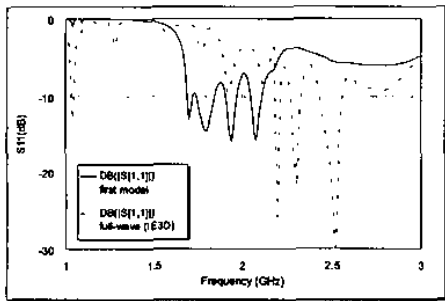

Fig. 4

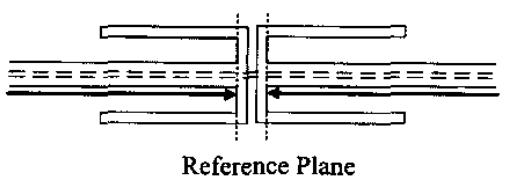

Fig. 5

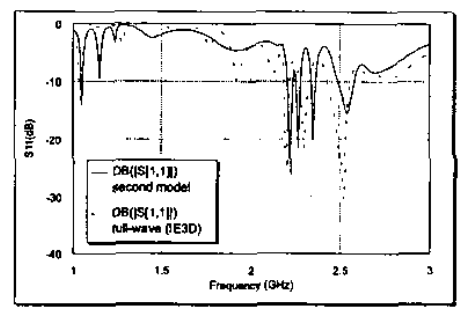

Fig. 6

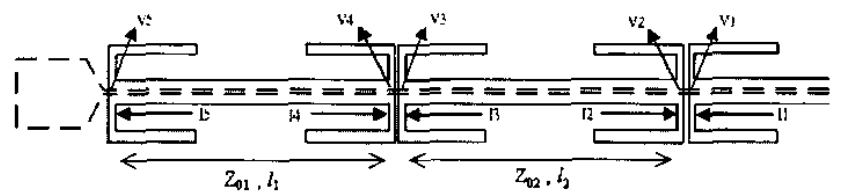

Fig. 7
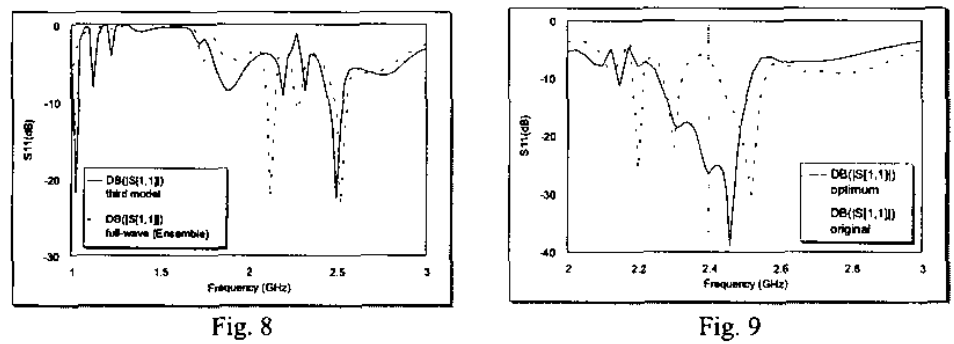

887 